\title{
Circuit
}

Musiques contemporaines

\section{L'après-rupture The afterbreak}

\section{Isabelle Panneton}

Volume 7, numéro 1, 1996

Ruptures?

URI : https://id.erudit.org/iderudit/902151ar

DOI : https://doi.org/10.7202/902151ar

Aller au sommaire du numéro

Éditeur(s)

Les Presses de l'Université de Montréal

ISSN

1183-1693 (imprimé)

1488-9692 (numérique)

Découvrir la revue

Citer cet article

Panneton, I. (1996). L'après-rupture. Circuit, 7(1), 29-33.

https://doi.org/10.7202/902151ar

\section{Résumé de l'article}

La compositrice se situe dans la continuité historique des créateurs qui ont répondu par leurs orientations nouvelles à l'érosion du système tonal, mais souligne que le défi de la musique d'aujourd'hui est d'offrir à l'auditeur des repères qui conduisent l'écoute aussi sûrement que le faisait le système tonal. À la notion de rupture, elle propose de substituer celles de transformations, de renouvellements et d'ouvertures et rappelle que, à côté des diverses fonctions qu'assume la musique aujourd'hui, la musique reste un art en soi.
Tous droits réservés @ Les Presses de l’Université de Montréal, 1996
Ce document est protégé par la loi sur le droit d'auteur. L’utilisation des services d'Érudit (y compris la reproduction) est assujettie à sa politique d'utilisation que vous pouvez consulter en ligne.

https://apropos.erudit.org/fr/usagers/politique-dutilisation/ 


\section{L'après-rupture} Isabelle Panneton

I Texte paru dans Le Devoir, 7 novembre 1994.

Le 3 octobre dernier, Lise Bissonnette publiait en page éditoriale du Devoir une chronique intitulée "Ruptures " que j'ai parcourue avec d'autant plus d'intérêt qu'il est assez rare que la musique classique, et plus particulièrement la musique contemporaine, occupe une telle place dans un quotidien de cette importance. Madame Bissonnette y soulève des problèmes de fond par rapport à la situation de la musique contemporaine au Québec tout en interpellant directement le milieu musical : "Y a-til une réponse dans la salle ? » $\|$ est impossible, pour une seule personne et en un seul article, de répondre de façon substantielle à ces questions, mais j'aimerais tout de même soumettre quelques éléments de réflexion. 
" Je comprends le drame des créateurs, qui vivent sous les ordres de la rupture, mais je ne les entends pas répondre ", écrit Madame Bissonnette.

\section{Sous les ordres?}

En tant que compositrice, je dois d'abord dire que je ne me sens pas tant "sous les ordres " d'une rupture (donc, dans l'obligation morale de faire radicalement nouveau, par exemple en cassant des assiettes sur une timbale), que dans la retombée des bouleversements du début du $x x^{e}$ siècle, dans une " aprèsrupture » qui laisse place à une incroyable liberté et, par conséquent, à une multiplicité de tendances: entre autres celle qui intègre les nouveaux moyens offerts par la technologie, celle qui renove avec des éléments de la tradition, celle encore qui puise son matériau dans des sources extrêmement variées, de la musique populaire à la musique ethnique en passant par le jazz et même la musique classique...

Cela dit, reconnaissons qu'à l'intérieur de ces nouveaux espaces, le défi de la musique d'aujourd'hui est de taille : il faut offrir à l'auditeur des repères, des lignes de force qui conduisent l'écoute aussi sûrement que le faisait le système tonal. Car, au-delà de tout débat esthétique, c'est bien là qu'elle se situe, la rupture " originelle » qui préside à toutes les autres... ce fameux système tonal, dont on parle comme s'il s'agissait d'un objet fixe que quelques esprits originaux, au début du $x x^{e}$ siècle, auraient décidé de « balancer " par-dessus bord. L'on tend à oublier que la tonalité est une organisation de la matière sonore qui s'est constituée lentement, au fil des siècles et des ruptures stylistiques, et qu'il y a un immense fossé entre les contrepoints de Palestrina à une extrémité et la polyphonie des postromantiques à l'autre. L'érosion du système tonal, l'épuisement des fonctions dominantes-toniques et de toute la hiérarchie qui lui donnait son intelligibilité furent profondément ressentis par des compositeurs tels que Claude Debussy et, d'une façon toute différente, par Schönberg, Webern et Berg. On ne peut que reconnaître, aujourd'hui, que leur démarche s'inscrit dans une continuité historique.

Je ne dis pas que le système tonal, à lui seul, assurait la cohérence d'une œuvre, mais bien qu'il occupait une place prépondérante, au cœur de l'articulation de tous les autres paramètres (mélodie, harmonie, rythme, timbre, consonances et dissonances). Or, le passage de la tonalité à l'atonalité, même s'il procède d'une logique, aura entraîné une remise en question, encore en œuvre aujourd'hui, de tous les aspects du langage sonore. La disparition du figuratif, dans la peinture du début du siècle, aura peut-être eu un effet comparable : à partir du moment où la référence que constituait la reproduction d'une image disparaissait, l'œuvre donnait à " voir " autre chose et permettait à tous les paramètres qui avaient été jusque-là assujettis à la figuration /couleurs, volumes, 
formes, etc.) d'avoir une vie propre et d'exprimer, de façon dramatique (les surréalistes) ou joyeuse (Miró), tous les replis de l'inconscient.

Une fois le langage musical dégagé du système tonal et des notions qui lui étaient associées (entre autres celle de mélodie), de nouvelles avenues se sont ouvertes dans l'exploration du timbre, du rythme, de la forme, et dans la constitution de textures et de modes inédits. Depuis les années 1950, les développements technologiques ont mis de nouveaux outils à la disposition des compositeurs et donné une impulsion extraordinaire à cette exploration. Plutôt que de ruptures, il s'agit donc de transformations, de renouvellements, d'ouvertures...

II n'en demeure pas moins qu'il faudrait peut-être redéfinir ce qui, au-delà des langages et des styles, a assuré de façon non équivoque l'intelligibilité du discours musical, non seulement dans les chefs-d'œuvre du $x^{\mathrm{e}}$ siècle, mais aussi dans ceux de toutes les époques... Et cette réflexion, les créateurs, les musicologues et les interprètes se doivent de la renouveler devant les gens à qui ils demandent d'accueillir leur travail : le public.

Pour retrouver la complicité de ce dernier, quoi qu'on en dise, la fréquentation des œuvres du $x x^{e}$ siècle demeure une question déterminante. J'aimerais citer à ce propos un commentaire de Milan Kundera :

[...] Jusqu'au xixe siècle, la société vivait presque exclusivement avec la seule musique contemporaine. Elle n'avait pas de contact vivant avec le passé musi$\mathrm{cal}$ : même si les musiciens avaient étudié (rarement) la musique des époques précédentes, ils n'avaient pas l'habitude de l'exécuter publiquement. C'est durant le xixe siècle que la musique du passé commence à revivre à côté de la musique contemporaine et à prendre progressivement de plus en plus de place, si bien qu'au $x x^{x}$ siècle, le rapport entre le présent et le passé se renverse : on écoute la musique des époques anciennes beaucoup plus qu'on écoute la musique contemporaine qui, aujourd'hui, a fini par quitter presque complètement les salles de concert. (Kundera, 1993, pp. 78-79)

Paradoxalement, les musiciens d'aujourd'hui peuvent passer leur vie à interpréter les œuvres du passé sans jamais assumer leur responsabilité, fût-ce occasionnellement, face au présent. Heureusement, la situation a beaucoup évolué depuis le début des années 1960, les interprètes qui intègrent la musique contemporaine à des programmes traditionnels sont de plus en plus nombreux et les « classiques » du $x^{*}$ siècle commencent à être interprétés de façon plus régulière. Ceux qui se consacrent à temps plein à la diffusion de la musique actuelle ont une lourde tâche : ils prennent le risque d'imposer en concert des œuvres récentes dont on ne connaît pas encore la valeur, en sachant que l'évolution d'un compositeur, et de la musique tout court, est directement liée à la réalisation sonore des partitions ( $c$ 'est d'ailleurs la première et la plus redoutable des critiques, croyez-moil. 


\section{La mémoire vive}

Sur un autre plan, il nous faut tenter de faire le poids avec la mémoire vive que constituent, auprès du public, les enregistrements des œuvres traditionnelles qui favorisent d'emblée l'assimilation des musiques les plus complexes, des styles les plus variés. Comme on le sait, la musique contemporaine est fort peu endisquée et, lorsqu'elle l'est, elle ne reçoit à peu près aucune couverture médiatique. Á preuve cette mauvaise habitude qu'ont les critiques de présenter une quinzaine de nouveaux enregistrements produits au Québec (de musique traditionnelle et contemporainel en des espèces d'articles-paniers qui occupent... une demi-page de journal, alors qu'ils consacrent facilement un article complet à une production venue de l'extérieur. Manque de place dans nos quotidiens ? Manque de compétence pour appréhender, cerner, situer ce qui vient d'ici, ce qui est nouveau ? Y a-t-il une réponse dans la salle de rédaction?

Dans la lancée des développements technologiques, la musique a envahi tous les secteurs de notre vie quotidienne et, pour le meilleur et pour le pire, on lui demande d'assumer diverses «fonctions": dans les films, dans les centres commerciaux et les métros, pour faire relaxer dans des thérapies de toutes sortes... Si ces applications ont leur place, elles ne doivent pas nous faire oublier que la musique est un art en soi, un art qui se donne à entendre et dont le déploiement dans le temps et dans l'espace requiert un minimum d'attention, ce que l'on pourrait appeler une écoute active. Essayez donc de découvrir un quatuor à cordes de Beethoven en musique de fond... Insupportable !

J'aimerais commenter l'affirmation selon laquelle le système d'art qu'est la musique contemporaine se construirait "entièrement, volontairement, hors de nous ». Jamais, au fil de ma démarche, je n'ai fait le choix de composer une musique marginale : je suis depuis toujours à la merci de mes idées, aux prises avec un langage qui me travaille de l'intérieur, avec des éblouissements et des contradictions qui me viennent de partout et en cela, j'ai le sentiment d'être en phase avec mon époque.

\section{Postmortem}

L'article de Lise Bissonnette aura donc suscité un débat où des considérations de toutes sortes ont émergé, et ce de la part d'intervenants issus de divers milieux. Je demeure un peu saisie de la façon dont la musique contemporaine s'est trouvée soumise à la question de la rentabilité : elle ne rapporte pas parce qu'elle ne rejoint pas un large public, donc les compositeurs doivent modifier leur approche et remettre leur démarche en question. Nombreux furent les propos, inscrits dans le sillage de la marchandise à vendre au plus grand nombre, où se profilait une dangereuse équation : rentabilité $=$ accessibilité $=$ preuve 
de la qualité d'une œuvre, "somme toute "! II y aurait encore tellement à dire sur chacun des termes en présence...

Mentionnons seulement que la musique est un « objet » susceptible de profiter au maximum du réseau des nouvelles techniques de communication. Paradoxalement, on le sait, l'extraordinaire circulation de toutes les musiques (savantes, populaires, d'hier et d'aujourd'hui, d'ici et d'ailleurs), toute enrichissante qu'elle soit, comporte en elle-même un risque réel: celui de produire un effet "varlope $e^{(1)}$ ", un aplanissement des différences. Les nombreux acteurs de la musique contemporaine (des interprètes aux diffuseurs) n'en sont que davantage soumis à la tyrannie de la rentabilité, une tyrannie qui exclut tout sens critique face aux enjeux réels de la démarche créatrice.

Fautil répéter que l'art (tout court) - comme la philosophie, la poésie ou toute forme de recherche - n'a pas à rejoindre un large public pour être essentiel au développement d'une société ? Et si, au-delà d'un débat esthétique, l'une des réussites de la musique contemporaine était ce que d'aucuns perçoivent comme son échec ? C'est-à-dire un refus, conscient ou non, de revêtir des atours qui en feraient un objet attrayant auquel nul ne saurait résister, bref, un produit que l'on évaluerait selon sa rentabilité sur le marché de la consommation.

J'ai la naiveté de croire que le prétendu problème-de-l'accessibilité-de-lamusique-contemporaine témoigne en fait d'une vitalité, d'un esprit de recherche et d'ouverture et, ultimement, d'un espace de résistance qui produit un retournement de toutes les questions...
(1) "Varlope " est une expression populaire québécoise utilisée pour désigner un rabot. (NDIR) 